\title{
ANGULAR DEPENDENCE OF HIGHLY NONLINEAR PULSE SPLITTING IN A TWO DIMENSIONAL GRANULAR NETWORK
}

\author{
D. Ngo \\ Aeronautics (GALCIT) \\ California Institute of Technology \\ Pasadena, CA, USA
}

\author{
F. Fraternali \\ Department of Civil Engineering \\ University of Salerno \\ Fisciano (SA), Italy
}

\author{
C. Daraio \\ Aeronautics (GALCIT) \\ Department of Applied Physics \\ California Institute of Technology \\ Pasadena, CA, USA
}

\section{ABSTRACT}

We investigate experimentally and numerically the propagation of highly nonlinear signals in a branched twoJinensional granular system composed by chains of uniform spherical beads. The system consists of a Y-shaped guide with various branch angles in which stainless steel spheres are arranged. We study the dynamic behavior of a solitary pulse irossing the bifurcated interface, and splitting between the two branches. We report for the first time the dependence of the split pulses' speed on the branch angles. Numerical simulations based on Hertzian interaction between the particles are found in agreement with the experimental data.

\section{INTRODUCTION}

The propagation of strongly nonlinear solitary waves in one-dimensional systems composed of uncompressed spherical particles has been recently receiving increasing attention [117]. A peculiar property of these systems is the tunability of wave propagation, which can be obtained by changing the material and the geometrical properties of the beads, and the static precompression $[1,3,4,6]$. Extensive theoretical, experimental and numerical studies have been conducted for uniform and heterogeneous one-dimensional systems [1-17]. Such systems have been proposed for a variety of engineering applications, including energy trapping and impulse disintegration $[8,9,13,17]$. Two-dimensional systems composed of spherical particles arranged in a Y-shaped configuration were also investigated $[18,19]$. In these systems, a symmetric $Y$ shaped guide was used to analyze the propagating of single and multiple highly nonlinear solitary waves, in both uniform and heterogeneous arrangements of particles. Solitary waves were demonstrated capable of following a bent trajectory, and could split after crossing the bifurcated interface. Arrangements of particles composed of heterogeneous materials were also shown capable of trapping and delaying pulses [18,19].

In this paper, we conduct numerical and experimental studies on the propagation of a single solitary wave in systems with variable angles between the two branches. We study the tunability of the solitary wave speed as a function of the system's geometry. These studies could be utilized to design new materials capable of redirecting stresses and impulses and to understand the angular dependence of dynamic load transfer in two-dimensional granular material media [20].

\section{EXPERIMENTAL SETUP AND RESULTS}

We designed a set of Y-shaped guides with different bifurcated angles between the two branches as shown in Fig. 1, where $\alpha$ is the branch angle between the first branch and the main stem, $\beta$ is the branch angle between the second branch and the main stem. The guides were carved in polycarbonate blocks, and filled with identical stainless steel (type 440C) spherical particles of uniform diameter of $9.52 \mathrm{~mm}$ (15 particles in the main stem and 15 particles in each branch). At the interface, three chains (the stem and the two branches) share 
one particle to allow splitting the compressive pulse formed in the stem by an impact. The properties of the materials composing the beads and the guide used in this study are summarized in Table 1. Six calibrated piezo-sensors were embedded inside particles in the system as described in $[1,2]$ (see Fig. 1(b)) and connected to Tektronix oscilloscopes, allowing for calculating the signal's speed and monitoring the wave's shape before and after the Y-junction interface. The whole setup was tilted at small angle $\left(\sim 4^{0}\right)$ to ensure the contact among the particles. Single solitary waves were generated in the system by dropping an identical stainless steel bead as a striker from a ramp. The striker's impact velocity was measured using an optical velocimeter connected to a digital oscilloscope.

\begin{tabular}{|c|c|c|c|c|}
\hline & $\begin{array}{c}\text { Elastic } \\
\text { modulus } \\
{[\mathrm{GPa}]}\end{array}$ & $\begin{array}{c}\text { Poisson } \\
\text { ratio }\end{array}$ & $\begin{array}{c}\text { Density } \\
{[\mathrm{Kg} / \mathrm{m} 3]}\end{array}$ & $\begin{array}{c}\text { Bead } \\
\text { Mass } \\
{[\mathrm{g}]}\end{array}$ \\
\hline $\begin{array}{c}\text { Stainless steel } \\
\text { beads }\end{array}$ & 200 & 0.28 & 7800 & 0.45 \\
\hline $\begin{array}{c}\text { Polycarbonate } \\
\text { (wall) }\end{array}$ & 2.4 & 0.37 & 1220 & \\
\hline
\end{tabular}

Tab. 1: Properties of the materials composing the highly nonlinear systems tested experimentally.

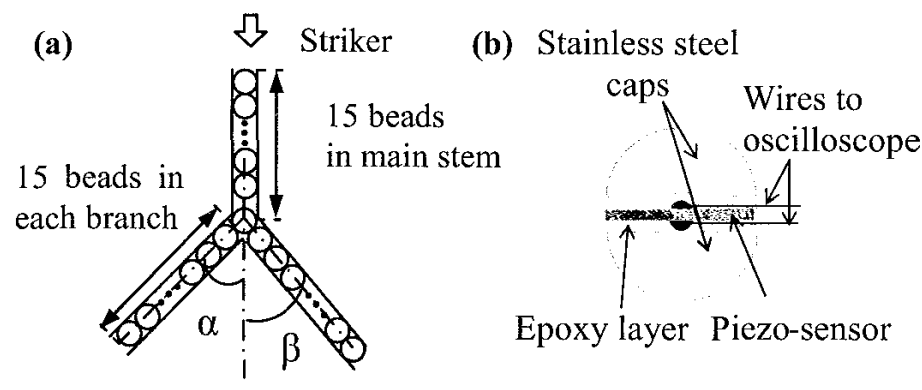

Fig. 1: (a) Experimental setup showing the Y-shaped guide and indicating the number of beads composing each portion of the system. (b) Schematic diagram of the composition of a particle with an embedded piezo-gauge $[1,2]$.

The first set of experiments was conducted on the symmetric Y-shaped systems, in which the branch angles were restricted to the case $\alpha=\beta$. Tests were performed for different values of branch angles $\left(\alpha=\beta=30^{\circ}, 35^{\circ}, 40^{\circ}\right.$ and $\left.45^{\circ}\right)$. The experimental results obtained for the different cases are shown in Fig. 2. The striker velocity used to generate the incoming solitary wave before the split interface was the same for all cases $(0.56 \mathrm{~m} / \mathrm{s})$. Six instrumented particles with sensors were inserted in the following positions: $10^{\text {th }}$ and $13^{\text {th }}$ particle in the main stem (counting from the striker side), and $5^{\text {th }}$ and $8^{\text {th }}$ particles in the two branches (counting from the interface), as pointed by the arrows in the corresponding curves in Fig. 2. From these results we observed that for all $\alpha=\beta$ angles tested, the incident solitary wave crossed the Y-junction interface and split into two identical single solitary waves traveling in the branches. This was also shown for the $30^{\circ}$ case in [19]. In addition, a reflected solitary wave could be observes propagating backward from the Y-junction in the main stem, shown in Fig. 2 (refer to the small amplitude pulses evident the top two lines, reporting the signals detected in the stem). The experimental results showed that the amplitude an speed of the reflected wave decreased when the branch angle increased. In the case of $\alpha=\beta=45^{\circ}$, no reflected wave in the main stem was observed in the experiments.
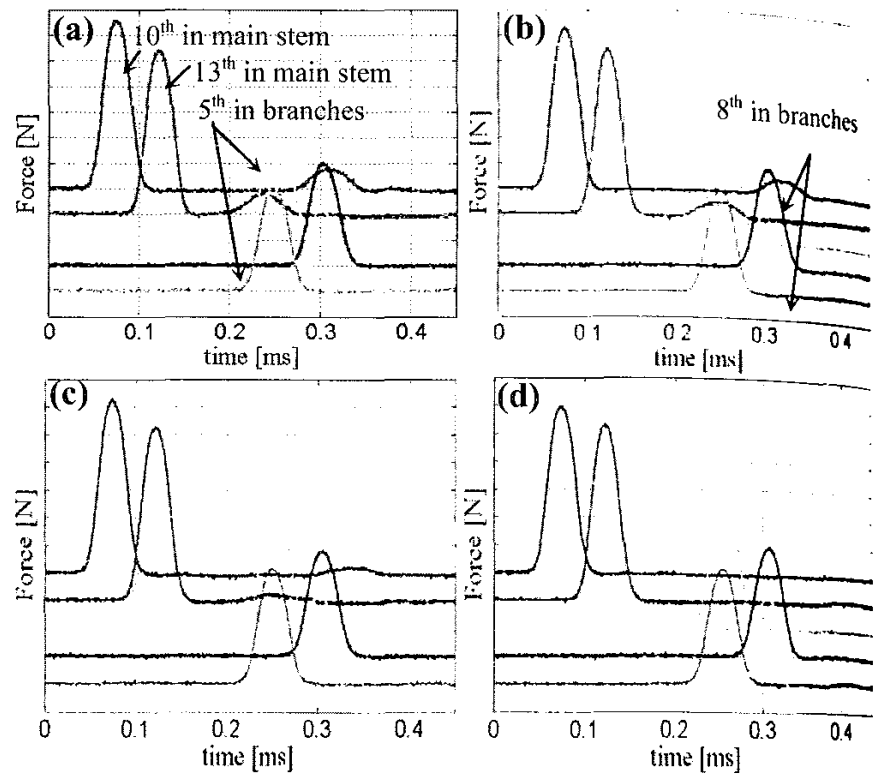

Fig. 2: (Color online) Experimental results for single solitary waves propagating in uniform, symmetric Y-shaped systems composed of stainless steel beads. (a) $\alpha=\beta=30^{\circ}$. (b) $\alpha=\beta=35^{\circ}$. (c) $\alpha=\beta=40^{\circ}$. (d) $\alpha=\beta=45^{\circ}$. The $y$-axes scale for all the curves is $10 \mathrm{~N}$ per division. The striker was dropped from the same height in all cases and the strike velocity was $0.56 \mathrm{~m} / \mathrm{s}$.

Experiments were performed also for uniform, asymmetri Y-shaped systems $(\alpha \neq \beta)$ in which the branch angle $\alpha$ was fixed at $\alpha=30^{\circ}$, and the angle $\beta$ was let to vary from $30^{\circ}$ to $60^{\circ}$. Results are shown in Fig. 3. It is evident that, similarly to what detected in the symmetric systems, single solitary wave: formed and traveled in the two branches. Also, a reflectei solitary wave was observed forming and traveling in the mair stem, after the incoming pulse interacted with the interface. Ths amplitude and speed of the wave reflected in the stem decreased with increasing branch angles. Differently than in the case of symmetric Y-shaped systems, the case $\alpha \neq \beta$ show asymmetry in the amplitude and speed of the transmitted waves propagating in two branches. We observed that the transmitted wave in the branch with the smaller branch angle ( $\alpha$ in this case) has larger amplitude and faster speed of propagation than the one in the other branch. 

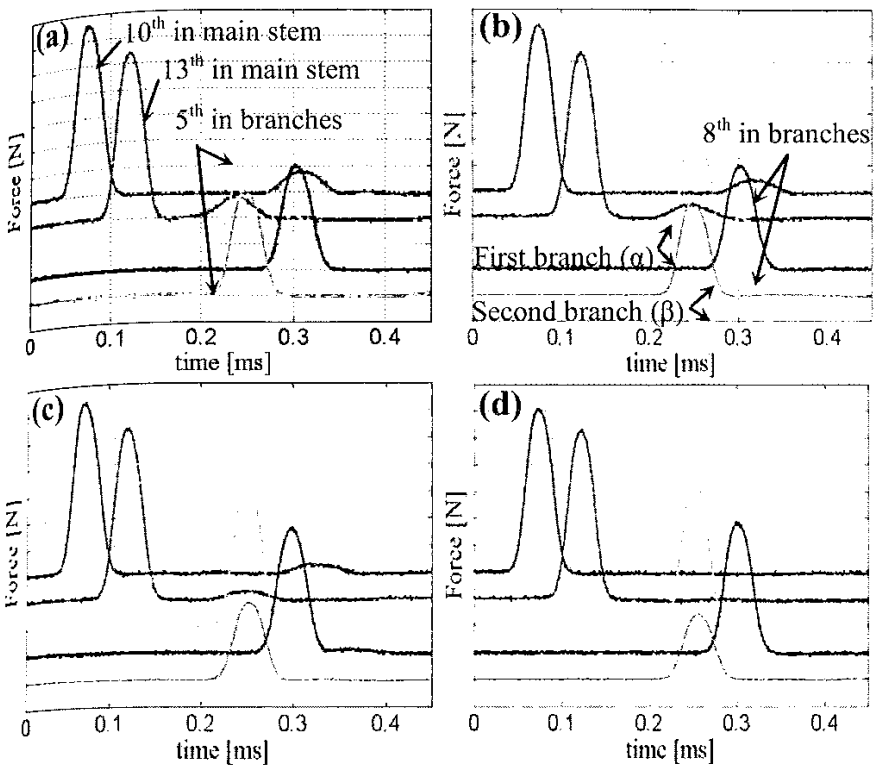

Fig. 3: (Color online) Experimental results for single solitary waves propagating in uniform, asymmetric Y-shaped systems composed of stainless steel beads. (a) $\alpha=30^{\circ}, \beta=30^{\circ}$. (b) $\alpha=30^{\circ}, \beta=40^{\circ}$. (c) $\alpha=30^{\circ}$, $\beta=50^{\circ}$. (d) $\alpha=30^{\circ}, \beta=60^{\circ}$. The y-axes scale for all the curves is $10 \mathrm{~N}$ per division. The striker was dropped from the same height for all the cases and the striker velocity was $0.56 \mathrm{~m} / \mathrm{s}$.

\section{NUMERICAL STUDY}

Numerical simulations were performed using the discrete numerical model described in papers $[17,19]$. Particles were treated as rigid bodies connected by nonlinear springs according to Hertzian contact laws between spheres. The walls of the Y-shaped guides were modeled as particles with infinite radii. The material parameters used in the numerical calculations, to model the particles used in experiments, are listed in Tab. 1. Dissipation was not included in the numerical simulations.

\section{DISCUSSION}

To characterize the behavior of single solitary waves crossing different Y-junction geometries, we compared the ratios of transmitted wave speeds (for pulses traveling in the branches after the interface) and reflected wave speeds (for pulses traveling back in the main stem after interacting with the interface) with the incident wave speeds. We indicate with $V_{s w}^{(t)}$ the wave speed of the pulses traveling in the symmetric branches. Similarly, we label $V_{s w}^{(t \alpha)}$ and $V_{s w}^{(t \beta)}$ the wave speed in the asymmetric branches, referring to the branch with angle $\alpha$ and $\beta$ respectively. The speed of the reflected wave traveling back in the stem is indicated as $V_{s w}^{(r)}$, while the incident wave speed is referred to as $V_{s w}^{(i)}$. We compare numerical results with experiments in Figs. 4, 5 and 6 , as a function of the different branch angles. In this case the error bars in the experimental data were calculated as the standard deviations of the data obtained from 5 repeated measurements with the same striker velocity for each set of branch angles.

Results show an excellent agreement between the experiments and the numerical calculations. In the symmetric Y-shaped system (Fig. 4), we observed that, for the same incident wave speed, the reflected wave speed decreases as the branch angles increase, while the transmitted wave speed shows a reverse dependence on branch angles. It should be pointed out that in the case $\alpha=\beta=45^{\circ}$ the numerical simulation showed the presence of a small reflected wave traveling back in the main stem. However, its small amplitude $(\sim 0.5 \%$ of the incident solitary wave amplitude) was not detectable in experiments.
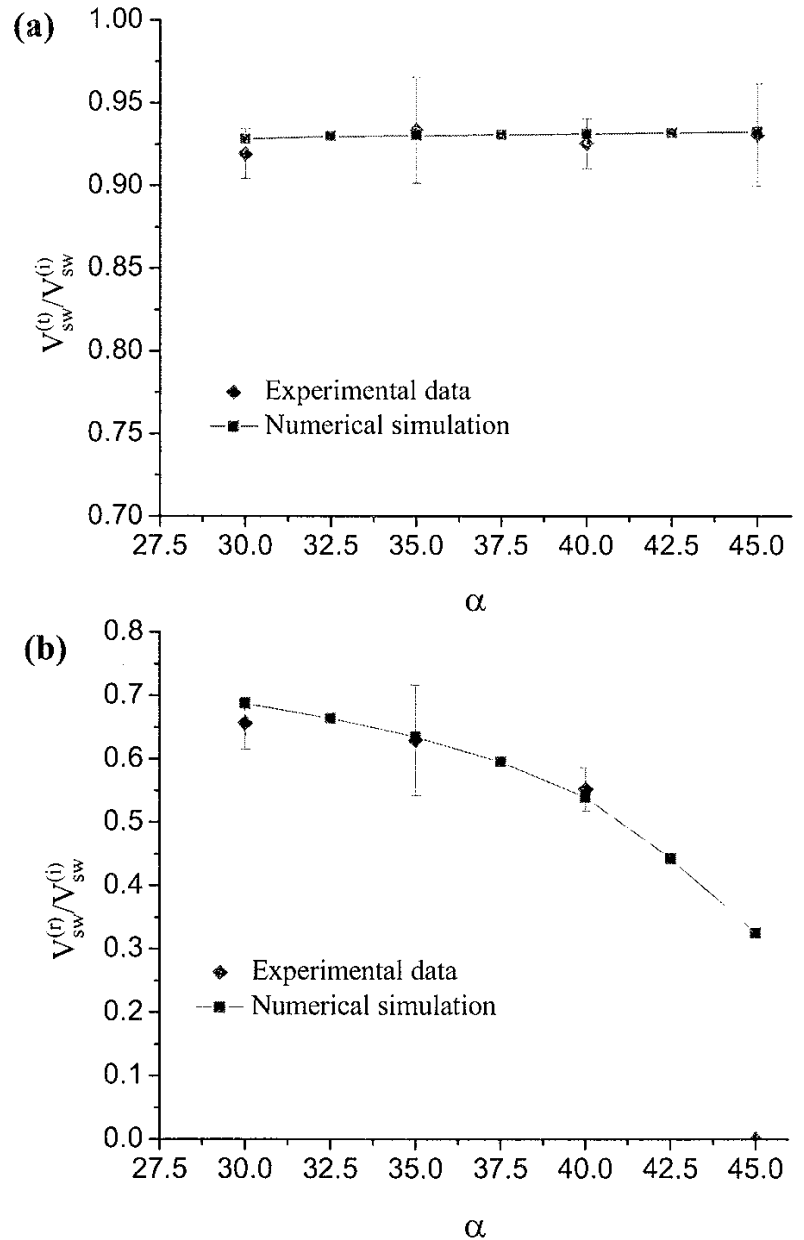

Fig. 4: Dependence of ratios of solitary wave speeds on the branch angles in the symmetric Y-shaped system $(\alpha=\beta)$. (a) Comparison of the experimental values with numerical results for $V_{s w}^{(t)} / V_{s w}^{(i)}$. (b) Comparison of the experimental values with numerical results for $V_{s w}^{(r)} / V_{s w}^{(i)}$. The striker velocity using in numerical calculations was $0.56 \mathrm{~m} / \mathrm{s}$.

Results obtained for the asymmetric Y-shaped systems for the specific case when $\alpha$ is fixed at $30^{\circ}$ and $\beta$ varies from $30^{\circ}$ to $60^{\circ}$ are reported in Figs. 5 and 6 . Due to the asymmetry of the 
two branches, the transmitted wave speeds $V_{s w}^{(t \alpha)}$ and $V_{s w}^{(t \beta)}$ in the branches now show different dependences on the branch angles. Figs. 5(a) and 5(b) show that for the same incident wave speed when the angle $\beta$ increases $(\beta \geq \alpha), V_{s w}^{(t \alpha)}$ increases while $V_{s w}^{(t \beta)}$ decreases. It also means that the difference between transmitted wave speeds in two branches represented by $V_{s w}^{(t \beta)} / V_{s w}^{(t \alpha)}$ depends on the difference between two branch angles and this is shown clearly in Fig. 5(c). As $\beta$ increases from $30^{\circ}$ or the difference between $\beta$ and $\alpha$ increases because of fixed $\alpha=30^{\circ}$, the ratio $V_{s w}^{(t \beta)} / V_{s w}^{(t \alpha)}$ decreases from unity. The relation of reflected wave speed $V_{s w}^{(r)}$ to the branch angles in the asymmetric systems shown in Fig. 6 is similar to the corresponding one in the symmetric systems (Fig. 4(b)). It should be noted that, in the case $\alpha=30^{\circ}, \beta=60^{\circ}$, the reflected wave was also observed in numerical simulations and it travelled in the main stem with very small amplitude that was undetectable in experiments.

The amplitude (maximum force, $F_{m}$ ) of the highly nonlinear solitary waves forming in this systems is related to the wave speed as $F_{m} \sim\left(V_{s w}\right)^{6}[1]$. The solitary wave amplitude ratios are expected to have similar dependence on the branch as the solitary wave speed ratios analyzed above. For example, Fig. 7 presents the experimental data and numerical calculations of the amplitude ratio $F_{m}^{(t \beta)} / F_{m}^{(t \alpha)}$ of two transmitted solitary waves in the branches with respect to the angle $\beta$. Comparing these results to Fig. 5(c), one can see that force amplitudes and velocities have a similar behavior with respect to variations of the angle $\beta$. The angular dependence of speed and amplitude of the solitary waves in the Y-shaped systems described in Figs. 4, 5 and 6 can be explained quantitatively by using quasi-particle model $[1,14]$ and the detailed analysis will be presented in future publications.

The results reported on the solitary wave interaction with the Y-shaped interface, and the subsequent bending of the pulse transmitted to the branches, show that it is possible to control the propagating pulse amplitude and speed in a twodimensional system. By changing the respective angles $\alpha$ and $\beta$, it is possible to redirect stress waves along different paths and control the signal amplitude and speed in the different directions. The energy $E$ and the momentum $I$ carried by the single solitary wave are proportional to the wave speed as $E \sim\left(V_{s w}\right)^{10}, I \sim\left(V_{s w}\right)^{5}[14]$, hence by using the asymmetric Yshaped system we can control the amount of the energy and the momentum carried by the incident wave transferred to each branch. This could be advantageous for the design of acoustic delay lines, vibration absorption and novel shock protecting systems.
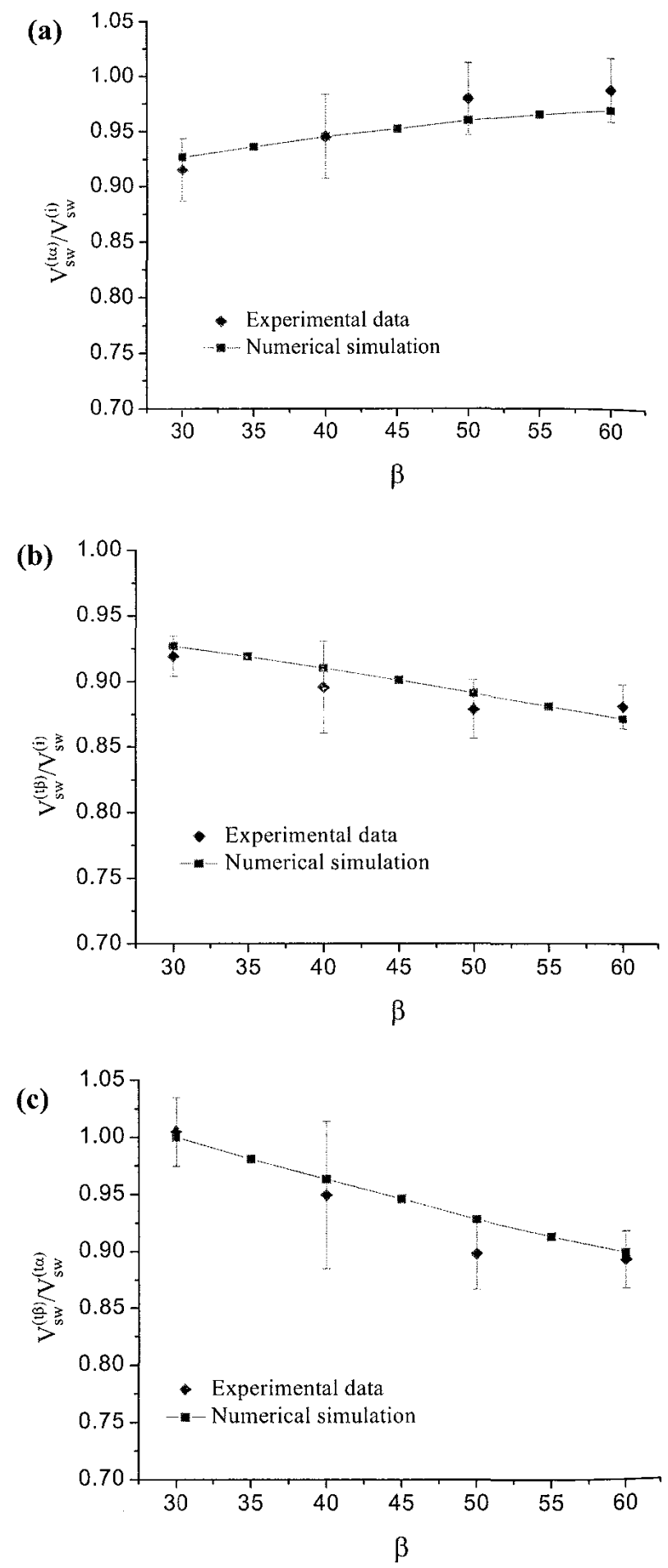

Fig. 5: Dependence of ratios of solitary wave speeds on the branch angles in the asymmetric Y-shaped system $(\alpha \neq \beta)$. (a) Comparison of the experimental values with numerical results for $V_{s w}^{(t \alpha)} / V_{s w}^{(i)}$. (b) Comparison of the experimental values with numerical results for $V_{s w}^{(t \beta)} / V_{s w}^{(i)}$. (c) Comparison of the experimental values with numerical results for $V_{s w}^{(t \beta)} / V_{s w}^{(t \alpha)}$. The angle $\alpha$ was fixed at $30^{\circ}$. The striker velocity using in numerical calculations was $0.56 \mathrm{~m} / \mathrm{s}$. 


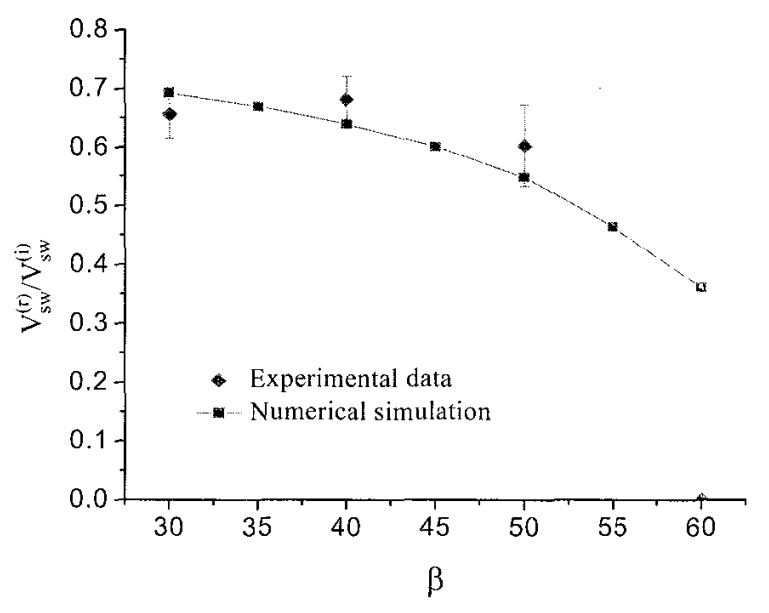

Fig. 6: The experimental and numerical results for the dependence of ratios of reflected wave speed and incident wave speed in the main stem $V_{s w}^{(r)} / V_{s w}^{(i)}$ on the branch angles in the asymmetric Y-shaped system $(\alpha \neq \beta)$. The angle $\alpha$ was fixed at $30^{\circ}$. The striker velocity using in numerical calculations was $0.56 \mathrm{~m} / \mathrm{s}$.

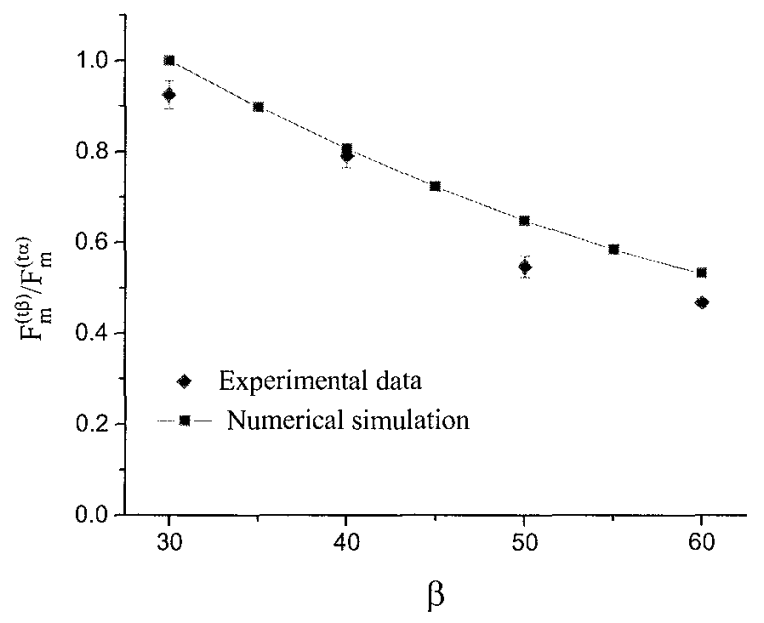

Fig. 7: The experimental and numerical results for the dependence of amplitude ratio of transmitted solitary waves $F_{m}^{(t \beta)} / F_{m}^{(t \alpha)}$ on the branch angles in the asymmetric Y-shaped systems. The angle $\alpha$ was fixed at $30^{\circ}$. The striker velocity using in numerical calculations was $0.56 \mathrm{~m} / \mathrm{s}$.

\section{CONCLUSIONS}

This paper describes the dynamics of single solitary waves propagating in Y-shaped systems with different branching geometries, using numerical and experimental approaches. We describe the wave speed variations as a function of the branch angles at the Y-junction and study the force amplitude of reflected and transmitted impulses in the different branches. We find excellent agreement between numerical and experimental data. The study of asymmetric Y-shaped systems showed the ability to redirect stress waves along different directions. The possibility to distribute different amounts of energy in the two branches, as a function of the branch angles, could be exploited in several practical applications.

\section{ACKNOWLEDGEMENTS}

Support for this work was received from the Army Research Office (MURI grant US ARO W911NF-09-1-0436, Dr. David Stepp and Proposal No. 54272-EG, Dr. Bruce LaMattina).

\section{REFERENCES}

1. Nesterenko, V. F., 2001, Dynamics of Heterogeneous Materials, Springer-Verlag, New York.

2. Nesterenko, V.F., 1983, "Propagation of nonlinear compression pulses in granular media", Journal of Applied Mechanics and Technical Physics, 24, pp. 733.

3. Daraio, C., Nesterenko, V.F., Herbold, E. B., Jin, S., 2005, "Strongly nonlinear waves in a chain of Teflon beads", Physical Review E, 72, 016603.

4. Daraio, C., Nesterenko, V.F., Herbold, E. B., Jin, S., 2006, "Tunability of solitary wave properties in one dimensional strongly nonlinear phononic crystal", Physical Review E, 73, 026610 .

5. Coste, C., Falcon, E., Fauve, S., 1997, "Solitary waves in a chain of beads under Hertz contact", Physical Review E, 56, pp. 6104-6117.

6. Coste, C., Gilles, B., 1999, "On the validity of Hertz contact law for granular material acoustics", European Physical Journal B, 7, pp. 155.

7. Rosas, A., Lindenberg, K., 2004, "Pulse propagation in chains with nonlinear interactions", Physical Review E, 69, 016615 .

8. Hong, J., 2005, "Universal power-law decay of the impulse energy in granular protector", Physical Review Letters, 94, 108001.

9. Doney, R.L., Sen, S., 2005, "Impulse absorption by tapered horizontal alignments of elastic spheres", Physical Review E, 72,

10. Nesterenko V. F., Daraio, C.,Herbold, E. B., Jin, S., 2005, "Anomalous wave reflection at the interface of two strongly nonlinear granular media", PhysicalReview Letters, 95, 158702.

11. Job, S., Melo, F., Sokolow, A., Sen, S., 2005, "How solitary waves interact with boundaries in a $1 \mathrm{~d}$ granular medium", PhysicalReview Letters, 94, 178002.

12. Daraio, C., Nesterenko, V.F., 2006, "Strongly nonlinear waves in a chain of polymer coated steel beads", Physical Review E,73, 026612.

13. Daraio, C., Nesterenko, V.F., Herbold, E. B., Jin, S., 2006, "Energy trapping and shock disintegration in a composite granular medium", Physical Review Letters, 96, 058002.

14. Job, S., Melo, F., Sokolow, A., Sen, S., 2007, "Solitary wave trains in granular chains: experiments, theory and simulations", Granular Matter, 10, pp. 13-20. 
15. Sen, S., Hong, J., Bang, J., Avalos, E., Doney, R., 2008, "Solitary waves in the granular chain", Physics Reports, 462, pp. 21-66.

16. Porter M.A., Daraio, C., Herbold, E. B., Szelengowicz, I., Kevrekidis, P.G., 2008, “ Highly nonlinear solitary waves in phononic crystal dimers", Physical Review E, 77, 015601 .

17. Fraternali, F., Porter, M.A., Daraio, C., 2010, "Optimal design of composite granular protectors", Mechanics of Advanced Materials and Structures, 17, pp. 1-19.

18. Daraio, C., Nesterenko, V.F., 2007, "Propagation of highly nonlinear signals in as two dimensional network of granular chains", APS - SCCM, AIP Conference Proceedings, Big Island, Hawaii, CP955, Edited by M. Elert, M.D. Furnish, R. Chau, N. Holmes and J. Nguyen, American Institute of Physics, pp. 1419.

19. Daraio, C., Ngo, D., Nesterenko, V.F., Fraternali, F., 2010, "Highly nonlinear pulses splitting and recombination in a two dimensional granular network", submitted.

20. Shukla, A., Zhu C.Y., Sadd, M., 1988, "Angular dependence of dynamic load transfer due to explosive loading in granular aggregate chains", Journal of Strain Analysis, 23, pp. 121-127. 